\title{
Hábito: Por que Devemos Estudá-lo e o que Podemos Fazer?
}

\author{
Fábio de Cristo \\ Universidade de Brasília e Centro Universitário de Brasília \\ Brasília, DF, Brasil \\ Hartmut Günther \\ Universidade de Brasília \\ Brasília, DF, Brasil
}

\begin{abstract}
RESUMO
Neste artigo, o hábito é apresentado como um conceito útil para tornar duradouros os comportamentos em prol do meio ambiente (e.g., usar transporte público). O hábito é um comportamento aprendido que, após ser repetido várias vezes, tornou-se automático, isto é, com pouca ou nenhuma deliberação do indivíduo. Alivia o esforço cognitivo do indivíduo de ponderar sempre os prós e contras das mais diversas situações. São comportamentos estáveis e difíceis de romper. Esta revisão aborda a formação e a manutenção do hábito, assim como são apresentadas quatro medidas psicológicas existentes. Ao final, discutem-se possíveis intervenções, utilizando-se o transporte como exemplo. As intervenções visam a incentivar a construção de novos hábitos que protegem o meio ambiente e a minimizar a força de hábitos antigos indesejados. Neste cenário, o hábito ganha relevância e pode ser mais uma ferramenta para enfrentar os desafios relativos à promoção de comportamentos ambientais relevantes.
\end{abstract}

Palavras-chave: Hábito; Automatismo; Comportamento pró-ambiental; Comportamento de viagem; Medida psicológica.

\section{ABSTRACT}

\section{Habit: Why Should We Study It and what Can We Do?}

In this article, the habit is introduced as a useful concept to make lasting pro-environmental behaviors (e.g., performing selective garbage collection, saving water and using public transportation). Habit is a learned behavior which, after being repeated several times, has become automatic, i.e., with little or no deliberation by an individual. It relieves the cognitive effort of an individual to always weigh the pros and cons of different situations. They are stable and hard-to-break behaviors. This review addresses the formation and maintenance of the habit, as well as presents four existing psychological measures. Finally, possible interventions are discussed, using transportation as an example. Interventions aim to encourage the formation of new habits that protect the environment and minimize the force of old unwanted habits. In this scenario, the habit gains relevance and can be one more tool to address the challenges related to the promotion of relevant environmentally-friendly behaviors.

Keywords: Habit; Automatism; Pro-environmental behavior; Travel behavior; Psychological measure.

\section{RESUMEN}

Hábito: ¿Por qué Estudiarlo y lo que Podemos Hacer?

En este artículo, el hábito se presenta como un concepto útil para realizar comportamientos proambientales duraderos (e.g., llevar a cabo la recogida selectiva, ahorrar agua y utilizar el transporte público). El hábito es un comportamiento aprendido que, después de ser repetida varias veces, se ha convertido en automático, i.e, con poca o ninguna deliberación por el individuo. Alivia el esfuerzo cognitivo de la persona que sopesar siempre los pros y los contras de las diferentes situaciones. Los comportamientos son estables y difíciles de romper. Esta revisión se refiere a la formación y mantenimiento del hábito, así como se presentan cuatro medidas psicológicas existentes. Finalmente, se discuten las posibles intervenciones, usando el transporte como un ejemplo. Las intervenciones dirigidas a fomentar la construcción de nuevos hábitos que protegen el medio ambiente y reduzcan al mínimo la fuerza de los viejos hábitos no deseados. En este escenario, el hábito se convierte en importante y puede ser una herramienta para hacer frente a los desafíos relacionados con la promoción de conductas de relevancia ambiental.

Palabras clave: Hábito; Automatismo; Comportamiento pro-ambiental; Comportamento de viaje; Medida psicológica. 
Estudos sugerem que cerca de $45 \%$ do nosso comportamento tende a repetir-se no mesmo ambiente físico, quase todos os dias (Wood, Quinn, \& Kashy, 2002). Esses comportamentos feitos repetidamente ocorrem sem pensarmos tanto, isto é, com pouca ou nenhuma intenção. Não raro, eles se tornam automatismos que nos ajudam a lidar com as demandas do dia a dia. Em consequência, todo o esforço para influenciar a intenção de comportamento poderá ser em vão devido às peculiaridades inerentes a esses comportamentos, dentre elas, serem realizados frequentemente em situações estáveis (Klöckner \& Verplanken, 2012; Verplanken, Myrbakk, \& Rudi, 2005). Por exemplo, mesmo querendo colaborar na coleta seletiva do condomínio, o indivíduo habituado a não fazê-lo, dificilmente separará adequadamente o lixo, mesmo com a divulgação no condomínio; ou ainda, mesmo que o indivíduo trabalhe perto de casa e que motive-se a ir a pé, ele, por força do hábito, dificilmente deixará seu automóvel em casa.

Neste artigo, abordaremos um tipo de automatismo: o hábito. Buscaremos responder às seguintes perguntas, cujos subtópicos configuramse nos critérios de escolha da literatura analisada: Em, por que devemos estudá-lo?, analisaremos o conceito, a formação e a manutenção do hábito, assim como as medidas psicológicas existentes. Em, o que podemos fazer?, o foco será a análise da natureza das intervenções e as possibilidades de ações, sendo que algumas possíveis aplicações de mudança e construção de novos hábitos serão ilustradas a partir do uso do transporte. Por meio da análise de artigos científicos, publicados nos principais periódicos internacionais, nós sumarizaremos alguns dos principais conhecimentos produzidos e ofereceremos um panorama atual das pesquisas sobre hábito nos tópicos abordados. Assim, queremos estimular estudos e intervenções com base nesse construto, para ajudar a superar os desafios no contexto brasileiro e latino-americano, onde os estudos sobre o hábito, na perspectiva que abordaremos aqui, estão em seu estágio inicial (Cristo, 2013).

\section{POR QUE DEVEMOS ESTUDAR O HÁBITO? CONCEITO E MEDIDAS PSICOLÓGICAS}

Hábito é um comportamento aprendido que, após ser repetido várias vezes, tornou-se automático, isto é, com pouca ou nenhuma deliberação do indivíduo. A importância deste automatismo para o ser humano é a sua utilidade para obtermos algum resultado ou objetivo sem tomarmos novas decisões o tempo todo, e por aliviar o esforço cognitivo do indivíduo de ponderar sempre os prós e contras das mais diversas situações (Verplanken, 2010; Verplanken \& Aarts, 1999; Verplanken et al., 1998). O hábito e a intenção, portanto, interagem na predição de decisões posteriores (Gardner, de Bruijn, \& Lally, 2011). Assim, quando os comportamentos são realizados frequentemente e se tornam habituais, eles são menos guiados pela intenção e vice-versa, pois o hábito diminui a necessidade de obter informações para poder julgar e decidir. Por exemplo, Aarts, Verplanken e van Knippenberg (1997) evidenciaram que os ciclistas - pessoas fortemente habituadas a usar a bicicleta no dia a dia -, demoravam menos tempo para analisar uma situação e decidir se usariam a bicicleta (e.g., para ir para uma reunião na universidade) quando comparados com não ciclistas. Esses buscaram mais informação sobre a viagem e sobre outras opções de deslocamento, assim como ponderaram mais sobre a quantidade de esforço físico despendido e sobre o tempo de viagem.

Alguns autores (e.g., Ronis, Yates, \& Kirscht, 1989) sugerem que o comportamento torna-se habitual quando repetido frequentemente, pelo menos duas vezes ao mês, e extensivamente, pelo menos 10 vezes. Todavia, embora a história de repetição seja um dos elementos essenciais na formação do hábito, é difícil saber quantas repetições são necessárias para que o comportamento se torne, enfim, habitual, o que depende também da sua complexidade. Lally, van Jaarsveld, Potts e Wardle (2010), por sua vez, sugerem entre 18 e 254 dias de repetição, dependendo da complexidade do comportamento, tais como fazer uma alimentação saudável e realizar atividade física..

Mas, qual o processo psicológico associado à formação do hábito? Alguns autores (e.g., Abelson, 1981) sugerem que a repetição ajuda a formar roteiros mentais (ou scripts) que são recuperados da memória. O script é a representação mental de um conhecimento adquirido a partir de sequências de eventos que se repetiram. Desse modo, um script é ativado na memória quando estamos numa situação onde geralmente os eventos ocorrem em alguma sequência (Abelson, 1981). Com base nessa proposta, Gärling, Fugii e Boe (2001) apresentaram e testaram o Modelo do Processo de Aquisição de Escolhas Baseadas no Script que tenta explicar a formação do hábito, encontrando evidências favoráveis a essa ideia por meio de estudos correlacionais e experimentais. O modelo sugere a existência de duas fases, uma de "formação do script" e outra de "escolhas com base no script". A primeira fase pressupõe que as atitudes positivas, em conjunto com as experiências prévias do indivíduo, motivam o comportamento (neste caso, o uso do carro), desencadeando um processamento deliberado da informação em que se obtêm resultados positivos. 
A segunda fase se forma a partir da consolidação desse roteiro de escolha pela constante repetição, tornando-se acessível rapidamente na memória a partir dos estímulos situacionais. Gärling et al., (2001) sugerem, portanto, que a formação do hábito de usar automóvel foi consequência, inicialmente, da atitude positiva em relação ao ato de dirigir e, posteriormente, da consolidação dessas sucessivas escolhas em uma estrutura de conhecimento na memória (script). Assim, uma vez que esse script de usar o automóvel foi consolidado, as escolhas posteriores tenderam a ocorrer de maneira mais automática: o hábito havia se formado. Uma vez construído o hábito, é necessário que as circunstâncias continuem reforçando o script, i.e., sejam relativamente estáveis e que as sucessivas repetições sejam resultado de reforçamentos positivos (Aarts et al., 1998). Sem isso, a força do hábito tenderá a diminuir.

Em suma, o hábito é um construto psicológico que vai além da simples história de repetição do comportamento; é necessário que existam outras características, tais como, a automaticidade, a constância situacional e a funcionalidade. Na psicologia, historicamente, o hábito tem sido associado ao behaviorismo radical, que rejeitava a cognição como causa da ação e como mediadora das associações estímulo-resposta (Neal, Wood, \& Quinn, 2006). As perspectivas atuais, especialmente, relacionadas à cognição social, têm estudado o hábito a partir do que ocorre "dentro das pessoas". Esta concepção reflete o avanço teórico e empírico da literatura sobre este tema nos últimos 20 anos. Durante esse período, como o hábito tem sido mensurado? É o que apresentaremos na próxima subseção.

\section{MEDIDAS PSICOLÓGICAS DA FORÇA DO HÁBITO}

São apresentadas e analisadas, a seguir, quatro tipos ou formas disponíveis de mensuração do hábito: Frequência autorrelatada de comportamento passado, frequência autorrelatada do hábito, medida de frequência de resposta do hábito e índice de autorrelato do hábito. Essas medidas psicológicas têm sido usadas para medir vários hábitos, desde escolher um modo de transporte, comer frutos do mar, realizar atividade física até economizar dinheiro (e.g., Loibl, Kraybill, \& DeMay, 2011). A Tabela 1, elaborada com base em Verplanken et al. (2005), oferece uma síntese da análise dessas medidas psicológicas a partir dos seguintes critérios: propriedades psicométricas, clareza conceitual, vulnerabilidade a vieses e aspectos práticos (i.e, aplicabilidade e facilidade de uso).

\section{Frequência autorrelatada de comportamento passado}

É a operacionalização mais usada do hábito, além de ser a mais simples. Consiste em uma solicitação para que o respondente relate a frequência com que realiza um determinado comportamento-alvo, sendo usado apenas um item. Verplanken et al. (2005), por exemplo, operacionalizaram perguntando "quantas vezes o ônibus foi usado durante a semana passada". Klöckner, Matthies e Hunecke (2003), perguntaram aos participantes "o modo de transporte mais frequentemente usado para suas viagens ao centro da cidade", sendo o item acompanhado de uma escala de frequência.

Esta forma de mensuração, todavia, apresenta problemas, pois necessita que o participante recupere da memória exemplos de comportamentos passados. Tais lembranças podem não ser confiáveis, sendo propensas a vieses. Medidas psicológicas de autorrelato do hábito que são baseadas em apenas um item são propensas a sofrer de ausência de validade e precisão (Verplanken \& Orbell, 2003). Além disso, não cobrem um dos elementos importantes do hábito, a automaticidade.

\section{Frequência autorrelatada do hábito}

É uma pequena variação da medida anterior. Solicita-se ao participante que relate a frequência de um ato praticado no passado "sem consciência" ou "por força do hábito", explicitamente declarados no item. Nessa estratégia, além de buscar identificar a repetição (comportamento passado), tenta-se incorporar outra característica do hábito, ou seja, o aspecto não consciente da ação. Por exemplo, Wittenbraker, Gibbs e Kahle (1983) perguntaram "Quando você estava dirigindo automóvel, quantas vezes, nas últimas duas semanas, você colocou o cinto de segurança por força do hábito?” e Mittal (1988) operacionalizou por meio do seguinte item "Durante as últimas quatro semanas, quando eu entrei no meu automóvel, eu não estava consciente quando coloquei meu cinto de segurança", com uma escala de cinco pontos $(1=$ nunca; 5 =sempre $)$.

Os problemas dessa medida psicológica são semelhantes ao da frequência autorrelatada de comportamento passado. Adicionalmente, inclui duas informações em um único item, o que pode levar à confusão, isto é, o comportamento passado e a ausência de consciência. Considerando que lembrar pode ser uma tarefa difícil, dependendo do comportamento, decidir se essa ação foi ou não deliberada à época pode ser uma tarefa quase impossível. 
Convém recordar que os comportamentos habituais se caracterizam justamente por serem realizados sem muita deliberação (Verplanken \& Aarts, 1999). Por essas limitações, medidas alternativas foram desenvolvidas a partir da década de 1990.

\section{Medida de frequência de resposta do hábito (response frequency measure of habit-RF)}

Desenvolvida a partir de um programa de pesquisa sobre a escolha por um modo de transporte para deslocar-se no dia a dia (Verplanken et al., 1998), mas tem sido adaptada e usada em outros contextos (e.g., saúde). Ela mede a força do hábito, fundamentando-se na ideia de script que descrevemos no início da seção. Assim, por exemplo, quando um indivíduo usa habitualmente um modo de transporte para uma grande variedade de objetivos de viagem, esses objetivos, por sua vez, ao serem apresentados tornam-se capazes de ativar automaticamente o modo de transporte habitual (Verplanken et al., 1997).

Nesse caso do transporte, os itens do instrumento correspondem aos objetivos para realizar viagens, com a finalidade de se mensurar a automaticidade da escolha do modo de transporte entre elas. Por exemplo, Verplanken et al. (1997) apresentaram aos participantes do estudo nove viagens com objetivos específicos (e.g., "ir à praia com alguns amigos", "fazer um esporte como uma atividade de lazer", "fazer compras depois do trabalho"), assim como as opções disponíveis de deslocamento (a pé, ônibus, trem e bicicleta). A frequência das menções por um modo de transporte definido no estudo (no caso, bicicleta) entre os itens serviu para medir a força do hábito (Verplanken et al., 1997). Quando a força do hábito de usar a bicicleta aumenta, este modo tende a ser escolhido indistintamente em todos ou na maioria dos objetivos de viagem (itens). Inversamente, quando a força do hábito diminui, as pessoas podem exibir um padrão de mobilidade mais variável, ou ainda, ter um padrão de mobilidade em outro transporte que não a bicicleta. Em suma, de acordo com o RF, quanto maior a invariância das respostas, maior será o hábito.

Adicionalmente, durante a aplicação do RF, os participantes são submetidos a uma pressão de tempo por meio da presença do aplicador e da instrução para que mencionem, o mais rápido possível, a opção de resposta que vier à mente. O objetivo disso é reduzir a oportunidade de as pessoas deliberarem e, simultaneamente, promover respostas automáticas dirigidas por scripts.

Identificamos que as pesquisas variam quanto: ao número de itens (entre cinco e 15), às alternativas de resposta (entre quatro e sete, e.g., no caso do uso de transporte, carro, taxi, metrô, trem, à pé, ônibus e bicicleta, sendo que nem sempre todos são contemplados, o que depende do contexto da pesquisa); aos objetivos do comportamento e os contextos (e.g., a distância entre os destinos, no caso do transporte) (Aarts et al., 1997; Fujii \& Kitamura, 2003; Verplanken et al., 1998; Verplanken \& Orbell, 2003).

Klöckner et al. (2003) testaram quatro possibilidades de mudança em relação à medida original, a fim de aprimorá-la (e.g., possibilitar escolha de mais de um modo de transporte para cada viagem bicicleta e metrô -; mensurar o tempo de resposta de cada participante em cada item; descrever detalhadamente viagens e horários, em oposição às viagens com destinos e horários variados; incluir itens em terceira pessoa, em oposição à primeira pessoa usada no original). Todavia, os autores não obtiveram êxito. A medida original obteve melhores resultados do que a versão modificada, considerada parcimoniosa e coerente teoricamente.

Algumas críticas e limitações podem ser apontadas. O RF não é um instrumento padronizado; por isso, os itens são construídos/adaptados e pretestados a partir dos objetivos e especificidades de cada estudo, o que não a torna tão prática. Além disso, requer, preferencialmente, a presença de um aplicador para incentivar que as respostas sejam dadas rapidamente, sem tanta deliberação, e promover respostas mais automáticas. Todavia, o controle sobre o quanto as pessoas deliberam nessas respostas não é garantido e, com isso, as respostas podem expressar intenção em vez de hábito.

\section{Índice de autorrelato do hábito - IAH (self-report habit index - SRHI)}

É uma escala padronizada desenvolvida por Verplanken e Orbell (2003). Sua operacionalização baseia-se nas características do hábito como um construto psicológico além da repetição, como a dificuldade de controlá-lo, a falta de consciência e a eficiência. Inclui também a noção de que o hábito, de alguma forma, faz parte da identidade ou do estilo pessoal da pessoa, i.e, a pessoa considera que ela é o que ela faz habitualmente no cotidiano (Verplanken \& Orbell, 2003).

O instrumento é bastante conciso, com 12 itens, sendo três para cada característica, e possui o seguinte enunciado "O comportamento $X$ é algo que...", onde $X$ representa o comportamento que se quer estudar (e.g., usar o automóvel, separar os resíduos em casa ou economizar água). Posteriormente, são listados os itens, dentre eles: "eu faço frequentemente", "eu 
faço automaticamente", "eu faço sem ter que lembrar conscientemente". No original em inglês, a escala de respostas varia de 1 (concordo) a 7 (discordo). $\mathrm{Na}$ análise dos dados, os valores da escala são invertidos; assim, valores altos indicam hábitos fortes*. O IAH pode ser útil para estabelecer se determinado comportamento ganha ou perde características habituais, por exemplo, após uma intervenção para formar ou romper com hábitos indesejados (Verplanken \& Orbell, 2003).

Recentemente, IAH foi traduzido e adaptado para o contexto brasileiro. Cristo (2013) realizou dois estudos correlacionais com o IAH, sobre o hábito de usar automóvel, que objetivaram obter evidências de validade e de precisão do instrumento. Os resultados do Estudo 1, com 238 participantes, indicaram que o IAH obteve bons indicadores de precisão $(\alpha=0,95)$, de validade de construto (cargas fatoriais entre 0,74 e 0,86 , unidimensional) e de validade convergente com outras medidas de hábito: o RF, a frequência autorrelatada de comportamento passado (ambas $r=0,7, p=0,01$ ) e a quantidade de quilômetros rodados $(r=0,2, p=0,05)$. Os resultados do Estudo 2, com 970 participantes de vários estados brasileiros, por meio de um survey on-line, também evidenciou excelentes índices de precisão $(\alpha=0,95)$ e bons índices de validade de construto do IAH (cargas fatoriais entre 0,71 e 0,86). A validade convergente foi evidenciada por meio da correlação positiva com a frequência autorrelatada do comportamento passado para o uso do automóvel $(r=0,75, p<0,001)$ e a validade discriminante, por sua vez, foi evidenciada por meio de uma correlação negativa com o comportamento passado de usar ônibus $(r=-0,61, p<0,001)$.

Embora os aspectos positivos já apontados da IAH - i.e., a padronização, a brevidade e a fragmentação do hábito em componentes para facilitar a reflexão do respondente -, a medida pode não representar fielmente o comportamento. Está sujeita aos mesmos vieses inerentes às medidas de autorrelato. Além disso, não está claro na literatura o papel da "identidade" no hábito, aspecto incluído na medida. Apesar de ser potencialmente interessante conforme argumentado por seus autores, a identidade não tem sido descrita como uma característica do hábito em outras revisões (e.g., Verplanken \& Aarts, 1999; Verplanken et al., 2005). É necessário, ainda, maior clareza conceitual, uma vez que também é chamado de "estilo pessoal" ou "autoidentificação" (Cristo, no prelo).

TABELA 1

Síntese da Análise das Medidas Psicológicas da Força do Hábito (com base em Verplanken et al., 2005)

\begin{tabular}{|c|c|c|c|c|}
\hline $\begin{array}{l}\text { Critérios/ } \\
\text { Medidas }\end{array}$ & $\begin{array}{l}\text { Frequência autorrelatada } \\
\text { de comportamento passado }\end{array}$ & $\begin{array}{c}\text { Frequência } \\
\text { autorrelatada do hábito }\end{array}$ & $\begin{array}{c}\text { Medida de } \\
\text { frequência de resposta do hábito }-R F\end{array}$ & $\begin{array}{c}\text { Índice de } \\
\text { autorrelato do hábito }-I A H\end{array}$ \\
\hline $\begin{array}{l}\text { Propriedades } \\
\text { psicométricas }\end{array}$ & $\begin{array}{l}\text { Não se aplica. Apenas } \\
\text { um item. }\end{array}$ & $\begin{array}{l}\text { Não se aplica. Apenas } \\
\text { um item. }\end{array}$ & $\begin{array}{l}\text { Não se aplica. Consiste na contagem } \\
\text { do comportamento-alvo entre múltiplos } \\
\text { itens. }\end{array}$ & $\begin{array}{l}\text { Possui evidências de } \\
\text { confiabilidade interna e validade. } \\
\text { Múltiplos itens. }\end{array}$ \\
\hline $\begin{array}{l}\text { Clareza } \\
\text { conceitual }\end{array}$ & $\begin{array}{l}\text { Problemática. Nem todo } \\
\text { comportamento repetitivo é } \\
\text { habitual. }\end{array}$ & $\begin{array}{l}\text { Problemática. } \\
\text { Dificuldade de } \\
\text { interpretar: confusão } \\
\text { entre frequência e } \\
\text { força do hábito. }\end{array}$ & $\begin{array}{l}\text { Considera o hábito como construto } \\
\text { psicológico (tipo de comportamento } \\
\text { automático, direcionado para o objetivo). } \\
\text { Pressupõe que a escolha dominante por } \\
\text { uma opção deriva de scripts preexistentes, } \\
\text { refletindo o hábito. A pressão por } \\
\text { tempo diminui a possibilidade de se } \\
\text { fazer escolhas elaboradas, pesando, por } \\
\text { exemplo, prós e contras. }\end{array}$ & $\begin{array}{l}\text { Considera o hábito como } \\
\text { construto psicológico (tipo de } \\
\text { comportamento automático, } \\
\text { direcionado para o objetivo). } \\
\text { Mede as várias características } \\
\text { do hábito: história de repetição, } \\
\text { falta de controle, ausência de } \\
\text { consciência, eficiência mental e } \\
\text { identidade ou estilo pessoal. }\end{array}$ \\
\hline $\begin{array}{l}\text { Vulnerabilidade } \\
\text { a vieses }\end{array}$ & $\begin{array}{l}\text { Viés de julgamento } \\
\text { relacionado à precisão } \\
\text { da frequência relatada } \\
\text { de comportamento, } \\
\text { especialmente se não } \\
\text { for recente/saliente. }\end{array}$ & $\begin{array}{l}\text { Viés de julgamento } \\
\text { relacionado à precisão } \\
\text { da frequência relatada } \\
\text { de comportamento, } \\
\text { especialmente se não } \\
\text { for recente/saliente. }\end{array}$ & $\begin{array}{l}\text { Menos problemático no caso do } \\
\text { viés de julgamento da frequência de } \\
\text { comportamento. Problema potencial se } \\
\text { não for respondida } \\
\text { sob pressão, pois pode medir intenções/ } \\
\text { preferências em vez da força do hábito. }\end{array}$ & $\begin{array}{l}\text { Menos problemático no caso do } \\
\text { viés de julgamento da frequência } \\
\text { de comportamento. Inclui uma } \\
\text { característica ainda pouco } \\
\text { estudada do hábito: identidade } \\
\text { ou estilo pessoal. }\end{array}$ \\
\hline $\begin{array}{l}\text { Aspectos } \\
\text { práticos }\end{array}$ & $\begin{array}{l}\text { Pode ser autoadministrado } \\
\text { via questionário. Não requer } \\
\text { instruções específicas. }\end{array}$ & $\begin{array}{l}\text { Pode ser } \\
\text { autoadministrado via } \\
\text { questionário. Não } \\
\text { requer instruções } \\
\text { específicas. }\end{array}$ & $\begin{array}{l}\text { Necessidade de estudo piloto e preteste } \\
\text { para cada novo hábito/contexto. } \\
\text { Preferível não usar em questionário } \\
\text { autoadministrado: requerer pressão } \\
\text { por tempo. Usada em contextos onde } \\
\text { estão disponíveis múltiplas opções de } \\
\text { escolha de comportamento e/ou para } \\
\text { comportamentos que ocorrem em } \\
\text { situações variadas. }\end{array}$ & $\begin{array}{l}\text { Pode ser autoadministrado } \\
\text { via questionário. Não requer } \\
\text { instruções específicas. Potencial } \\
\text { para diferenciar as qualidades } \\
\text { habituais do comportamento. }\end{array}$ \\
\hline
\end{tabular}

\footnotetext{
* No Brasil, Cristo (2013) optou pela não inversão na análise, mas na própria escala de resposta, i.e., o respondente recebe a instrução de que $1=$ discordo fortemente e 7 = concordo fortemente, uma vez que, assim, haveria maior facilidade para responder do que ao contrário, de acordo com pretestes.
} 
Qual a melhor medida psicológica para fazer um estudo? Isso dependerá dos objetivos da pesquisa ou da intervenção, devendo ser balanceados os prós e os contras (veja um sumário na Tabela 1). Além do autorrelato, são possíveis alternativas metodológicas, tais como a observação continuada e os registros objetivos do comportamento. Na próxima seção, discutiremos implicações para a pesquisa e a intervenção no comportamento sob a perspectiva do hábito, recorrendo ao uso do automóvel como um exemplo. Iniciaremos com uma breve análise do contexto recente da mobilidade urbana no Brasil, para que se entenda a importância dessa intervenção.

\section{O QUE PODEMOS FAZER? \\ A REDUÇÃO NO USO DO AUTOMÓVEL COMO EXEMPLO DE INTERVENÇÃO}

De um lado, o contexto econômico brasileiro nos últimos anos (e.g., redução do Imposto sobre Produtos Industrializados - IPI) contribuiu para a compra e o uso frequente do carro no dia a dia, tornando-se um hábito para muitos. Isso pode ser evidenciado com o crescente número da frota de veículos e de viagens por esse modo de transporte. Por exemplo, estudos sugerem que o automóvel faz parte do padrão de comportamento de viagem de parcela dos brasileiros, aproximadamente $24 \%$ da população (Instituto de Pesquisa Econômica Aplicada [IPEA], 2011). Por outro lado, apesar dos benefícios trazidos com o seu advento, o automóvel tem sido considerado uma ameaça à qualidade de vida urbana por vários motivos, dentre eles, a poluição atmosférica, a poluição sonora e os acidentes de trânsito (Neto, Feitosa, Cristo, Cantal, \& Günther, 2013). Constitui-se, portanto, em um desafio para a psicologia, dentre outras áreas do conhecimento, desenvolver estratégias que tornem seu uso mais eficiente, reduzindo os congestionamentos, estimulando as pessoas a adotarem o transporte coletivo e/ou as alternativas menos poluentes como a bicicleta (Steg \& Vlek, 2009). Nesse contexto, o hábito tem sido considerado uma barreira importante para o desenvolvimento de alternativas de transportes sustentáveis, uma vez que impede a mudança de padrões de comportamentos já estabelecidos, danosos ao meio ambiente (American Psychological Association [APA], 2009; Steg \& Vlek, 2009).

A partir dos conhecimentos apresentados até aqui, é possível pensar em intervenções psicologicamente guiadas, a fim de contribuir com as pessoas na mudança/construção de hábitos de vida sustentável (e.g., caminhar e andar de bicicleta), e com os gestores de trânsito na implantação de medidas de gerencia- mento da demanda de tráfego, ou seja, na necessidade de usar automóvel. Como, então, a psicologia pode colaborar especificamente neste processo de mudança? As intervenções podem ser planejadas desde comportamentos mais simples aos mais complexos; desde a execução da manobra no veículo (interessantes para a segurança viária, como usar o cinto de segurança e o capacete, parar na faixa de pedestre) até a escolha do transporte (estimular as pessoas a andarem de bicicleta ou a pé).

Visando a definição das intervenções e a melhor maneira de implementá-las, deverá ser feito primeiramente uma análise e um diagnóstico para saber se os comportamentos-alvo são habituais e em que grau (veja seção anterior). As intervenções com base no hábito possuem especificidades, tendo em vista a pouca deliberação do indivíduo nas decisões (Verplanken, 2010). Mesmo algumas mudanças no ambiente poderão não ser suficientes para que o indivíduo as perceba, mantendo o seu comportamento habitual (Gärling et al., 2001). Uma vez constatadaa presença/ausência do hábito, existem pelo menos duas formas ou estratégias de intervenção, sendo que a escolha dependerá de alguns fatores e requererá cuidados específicos. Na primeira delas, o foco é incentivar a construção de novos hábitos; na segunda, o foco é tentar minimizar os hábitos já estabelecidos (Verplanken \& Wood, 2006).

\section{INCENTIVAR A CONSTRUÇÃO DE NOVOS HÁBITOS}

O processo de fazer com que um comportamento se torne hábito é um objetivo explícito de intervenção. Os novos comportamentos devem se tornar: frequentes, estáveis ao longo do tempo e resistentes a outras influências (Verplanken, 2010; Verplanken \& Wood, 2006). A lógica é influenciar as mesmas características que tornam os hábitos obstáculos, em favor do "novo" comportamento. Assim, tais características poderão ser consideradas desejáveis quando fizerem parte de um comportamento mais saudável e mais sustentável.

Seja para criar hábitos ou para mudar hábitos antigos indesejados, Verplanken (2010) argumenta que os indivíduos devem se sentir motivados para mudar ou adotar novos comportamentos. Um programa de intervenção, portanto, pode esperar bons resultados se os indivíduos tiverem, inicialmente, atitudes positivas e intenções fortes em relação ao comportamento novo. Reforçar este comportamento é importante para manter a sua repetição, como oferecer medidas de incremento dos benefícios de outros modos de transporte (e.g., 
melhorar o serviço de transporte coletivo e oferecer temporariamente passagens de ônibus gratuitas aos usuários habituais de automóveis; Fujii \& Kitamura, 2003). Todavia, tais medidas podem ser insuficientes por não serem percebidas pelas pessoas, posto que o hábito diminui a busca por informação (Fujii \& Gärling, 2007). Além disso, essa estratégia pode ser mais ou menos eficiente dependendo do modo de transporte. Por exemplo, Chen e Chao (2011) sugerem que os motoristas são menos influenciados por essas medidas do que os motociclistas porque, naqueles, o hábito exerce maior influência na intenção de mudar para o transporte público. Esse é um dado importante, pois demonstra o grau de influência do hábito (forte ou fraco) em diferentes categorias de habilitação e sugere uma alternativa para diminuir o aumento da frota de motocicletas, que seria mais propensa a mudar por meio de medidas não coercitivas (i.e., incentivos e educação), e diminuir o uso do automóvel por meio de medidas coercitivas (i.e, regulamentação de leis, pedágios e taxas para a circulação).

Embora a motivação seja necessária, ela é insuficiente. A pessoa pode não saber como agir, estar indecisa quando começar a se comportar ou, simplesmente, esquecer de fazer. Por isso, outro caminho possível para delinear e estabelecer novos hábitos é a partir da intenção de implementação. Significa criar um plano para transformar um objetivo (isto é, uma intenção) em uma ação concreta, ou seja, implementar algo que estamos motivados a fazer (Gollwitzer, 1999). Para elaborar um plano, é preciso selecionar a oportunidade mais adequada e os comportamentos mais efetivos para alcançar o objetivo almejado (e.g., usar o transporte coletivo). Especificamente, são definidos antecipadamente, nesse plano, três coisas: quando, onde e como implementar um objetivo. A seguinte frase ajuda a decidir esses pontos "Se a situação $\mathrm{X}$ acontecer, então eu farei Y" (Gollwitzer, 1999). O aspecto mais importante sobre um plano de implementação é a conexão dos estímulos específicos (quando; onde) com repostas específicas (o quê). Essa conexão deve se tornar forte. Essas novas respostas (e.g., ir de metrô para o trabalho), com o tempo, se tornarão tão automáticas quanto as respostas anteriores (e.g., usar o automóvel), substituindo-as. A tendência é que a resposta habitual anterior seja bloqueada. Dessa forma, é possível proteger com sucesso a busca pelo seu objetivo, ou seja, de se comportar de uma maneira mais sustentável. Formar intenção de implementação pode ajudar a estabelecer comportamentos que têm os elementoschave do hábito, formando um modelo cognitivo para o desenvolvimento de hábitos futuros.
Outra maneira, refere-se à socialização nos transportes (travel socialization), i.e., as crianças aprendem com os "agentes de socialização", por exemplo, pais e professores, sobre modos de viagem da mesma maneira que aprendem sobre a cultura. Haustein, Klöckner e Blöbaum (2009) sugerem que a comunicação com os pais sobre o impacto negativo de usar automóvel pode levar a formar um hábito fraco de usá-lo, sendo os filhos mais dispostos a usar modos alternativos. Essas intervenções também podem ser consideradas, em certo sentido, de prevenção da formação de hábitos danosos ao ambiente ou à saúde.

\section{MINIMIZAR A FORÇA DE HÁBITOS ANTIGOS}

O foco nessa estratégia é o comportamento "antigo", com o objetivo de minimizar ou romper com os hábitos já estabelecidos. Segundo Verplaken e Wood (2006), cabe eleger a intervenção entre dois grandes tipos, classificados por eles como:

1. Focadas em mudar comportamentos problemáticos ou indesejáveis por meio de educação, orientações, campanhas informativas e/ou programas de autoajuda (chamadas também de intervenções downstream). Uma variante desse tipo são aquelas onde as campanhas informativas ocorrem simultaneamente com a ocorrência natural de mudanças contextuais no estilo de vida do indivíduo, como mudança de residência e de local de emprego, tornando-o mais vulnerável às novas informações (ou downstreamPlus-Context-change). Neste caso, as mudanças ambientais, tanto físicas quanto sociais, dificultam a emissão de comportamentos habituais e ajudam a tornar as campanhas eficazes, porque o indivíduo estará mais "aberto" às novas informações para deslocar-se.

Alguns autores (e.g., Fujii \& Gärling, 2007) sugerem que as estratégias de comunicação são mais eficientes na fase de formação ou consolidação do script, isto é, quando o comportamento é repetitivo, mas, não necessariamente, um hábito ou quando é um hábito fraco. A comunicação pode ser direcionada para afetar alguns componentes importantes na formação do hábito, relacionadas às teorias: do comportamento planejado, i.e., atitude, controle percebido do comportamento, norma subjetiva e intenção de comportamento (Ajzen, 2011); da ativação da norma (obrigação moral); e da intenção de implementação.

A comunicação ainda poderá ser individualizada e/ou em massa (Fujii \& Gärling, 2007). Esta última, conforme os autores, é menos eficiente para romper o hábito do que a primeira, pois as mensagens podem 
ser ignoradas mais facilmente por tentar atingir um público maior, com mensagens mais gerais (e.g., propagandas de TV). Assim, a comunicação por meio do marketing individualizado, que provê informações personalizadas ao usuário do automóvel, seria mais eficiente, ou ainda a combinação com a comunicação em massa.

No Brasil, muito embora a política nacional de trânsito estabeleça prioridade aos modos de transporte mais frágeis e sustentáveis (como andar a pé), pouca ou nenhuma medida informativa (que tenha sido bem pensada e planejada) para desestimular o uso do automóvel foi adotada pelo governo federal ao longo dos últimos anos (Cristo, 2013). Ao contrário, em anos recentes, temos observado, tanto na mídia como nas ações políticas, o incentivo do governo à compra do automóvel e o subsídio à gasolina. Essa foi uma forma usada pelo governo, até o momento, para manter a economia brasileira aquecida para enfrentar as crises internacionais. As mudanças no padrão de compra de automóveis, a partir dos ajustes fiscais de 2015, deverão ser observados futuramente.

2. Focadas em promover contextos e estruturas sociais incompatíveis com o hábito que se quer modificar, ou seja, tenta mudar aquilo que sustenta o comportamento indesejado, seja por meio de desincentivos econômicos, legislação e/ou mudanças estruturais no ambiente, como as políticas públicas e o planejamento urbano (chamadas também de intervenções upstream). Fujii e Gärling (2007) sugerem as medidas de desincentivo aos benefícios associados ao uso do carro (push measures, e.g., cobrança de taxas e redução da capacidade das ruas). Em geral, elas possuem pouca aceitação das pessoas, especialmente quando não se tem um transporte coletivo de qualidade que dê suporte à obrigação de deixar o carro em casa.

Estratégias de comunicação são evitadas nesse tipo de intervenção. Mas, pode incluir, por exemplo, tentar diminuir congestionamentos por meio de solicitações na mídia para que não usem o automóvel para sair de casa em tal horário. São mais aceitas pela população, porém podem ser menos eficazes (Gärling, Gärling, \& Loukopoulos, 2002).

Verplaken e Wood (2006) integraram, junto a esses dois grandes tipos de intervenção, uma distinção entre hábitos fortes e fracos, produzindo diferentes abordagens de intervenções possíveis. Por exemplo, quando o comportamento a ser mudado possuir um componente habitual fraco ou inexistente, podem ser usadas intervenções downstream ou upstream para desencorajar um comportamento. Quando o hábito for forte, são recomendadas as intervenções downstream-Plus-Context-change ou upstream que modificam o ambiente.

Nos casos em que o hábito existir fortemente, é importante que os psicólogos e os responsáveis pela intervenção estejam atentos às dificuldades das pessoas (habituadas) em adaptar-se a tais mudanças, a fim de manejá-las. Além de ajudar as pessoas, tal acompanhamento reduz os riscos de a intervenção ser inviabilizada ou "não pegar" pelo sentimento de injustiça ou de revolta. Gärling et al., (2002) especularam sobre algumas consequências adversas esperadas para as pessoas se, no futuro, eles já não tiverem acesso livre aos seus carros, seja em função de uma escolha ou porque foram forçadas: (a) efeitos de curtoprazo - comprimir as atividades no tempo, mudar para modos de transporte mais lentos, aumento da combinação das atividades e coordenação das viagens; (b) efeitos intermediários - mudar de residência, de lugar de trabalho ou trabalhar horas através do teletrabalho; e (c) efeitos de longo-prazo - mudança de atividades de lazer, redução do bem-estar subjetivo e objetivo, mal funcionamento da família, estresse psicológico e fisiológico. Desse modo, as pessoas mais habituadas a usar carro poderão ter maior dificuldade do que as que não possuem tal hábito. Elas também poderão não aceitar as intervenções para gerenciamento da demanda de tráfego introduzidas ou em estudo de viabilidade técnica/política. Essas questões, todavia, carecem de respostas empíricas.

\section{SUMÁRIO E CONCLUSÕES}

Neste artigo discutimos o conceito de hábito, como se forma e se mantém, assim como as medidas psicológicas para avaliá-lo; em seguida, abordamos suas aplicações (neste caso, ilustrado no âmbito dos transportes). Vimos que as pesquisas sobre a relação atitude-comportamento, tem evidenciado que a atitude tem maior poder preditivo quando o comportamento é novo; todavia, quando o comportamento é frequente, do hábito aumenta o grau de predição do modelo (Bamberg \& Schmidt, 2003; Verplanken \& Aarts, 1999). Tais evidências empíricas são oriundas de estudos com variados métodos, tais como estudos de campo e meta-análises (Gardner, de Bruijn, \& Lally, 2011), e em vários domínios, desde consumir alimentos saudáveis, fazer compras, realizar atividade física, usar cinto de segurança até escolher um modo de transporte, o que sugere relevância em diversas situações, contextos e propósitos. A maioria das publicações é da Europa, da América do Norte e da Ásia. Não temos 
conhecimento, até o momento, de outros estudos que tenham abordado explicitamente o hábito, na perspectiva que tratamos aqui, no contexto latinoamericano (de modo geral) e brasileiro (de modo específico). Deve-se avançar nesse sentido.

O hábito, enquanto um comportamento estável e resistente à mudança ao longo do tempo, não é bom ou ruim por natureza. Sua funcionalidade permite que seja alvo das intervenções que visam inibi-lo, quando é considerado ruim ou danoso; e também pode ser estimulado, no caso da formação de novos hábitos, quando saudáveis ou respeitam o meio ambiente. Nesse sentido, discutimos a dificuldade de mudar o comportamento quando as pessoas estão habituadas (e.g., o de usar automóvel), devido a pouca deliberação sobre os prós e os contras nas decisões (e.g., de pegar o carro versus o transporte coletivo). Isso torna difícil o sucesso de intervenções com base em informação (e.g., propagandas incentivando o uso do ônibus), que são largamente usadas para mudar comportamento em geral, um investimento que pode ser em vão. Diante de tudo o que expomos no artigo, consideramos que o hábito tem ganho cada vez maior relevância e pode ser mais uma ferramenta para enfrentarmos os desafios relativos à promoção de comportamentos ambientais relevantes.

\section{REFERÊNCIAS}

Aarts, H., Verplanken, B., \& van Knippenberg, A. (1997). Habit and information use in travel mode choices. Acta Psychologica, 96, 1-14. http://dx.doi.org/10.1016/S0001-6918(97)00008-5

Aarts, H., Verplanken, B., \& van Knippenberg, A. (1998). Predicting behavior from the actions in the past: Repeated decision making or a matter of habit? Journal of Applied Social Psychology, 28(15), 1355-1374. http://dx.doi. org/10.1111/j.1559-1816.1998.tb01681.x

Abelson, R. P. (1981). Psychological status of the script concept. American Psychologist, 36(7), 715-729. http://dx.doi. org/10.1037/0003-066X.36.7.715

Ajzen, I. (2011). The theory of planned behavior. In P. A. M. van Lange, A. W. Kruglanski \& T. Higgins (Eds.). Handbook of theories of social psychology (Vol. 1, pp. 438-459). London: SAGE.

American Psychological Association. (2009). Psychology and global climate change: Addressing a multi-faceted phenomenon and set of challenges. Washington, DC: Autor.

Bamberg, S. \& Schmidt, P. (2003). Incentives, morality, or habit? Predicting students' car use for university routes with the models of Ajzen, Schwartz, and Triandis. Environment and Behavior, 35, 264-285. http://dx.doi. org/10.1177/0013916502250134

Chen, C. \& Chao, W. (2011). Habitual or reasoned? Using the theory of planned behavior, technology acceptance model, and habit to examine switching intentions toward public transit. Transportation Research Part F, 14(2), 128-137. http://dx.doi.org/10.1016/j.trf.2010.11.006

Cristo. F. (No prelo). Hábito e comportamento de viagem. In P. Taco, I. L. Neto, L. Santos, \& M. Takano (Eds.). Comportamento em transportes: uma abordagem multidisciplinar.

Cristo, F. (2013). O hábito de usar automóvel tem relação com o transporte coletivo ruim? (Tese de Doutorado). Retirado de: http://repositorio.unb.br/bitstream/10482/15413/1/2013_FabiodeCristo.pdf

Fujii, S. \& Gärling, T. (2007). Role and acquisition of car-use habit. In T. Gärling \& L. Steg (Eds.). Threats from the car traffic to the quality of the urban life: Problems, causes, and consequences (pp. 235-250). Amsterdam: Elsevier.

Fujii, S. \& Kitamura, R. (2003). What does a one-month free bus ticket do to habitual drivers? An experimental analysis of habit and attitude change. Transportation, 30, 81-95. http://dx.doi.org/10.1023/A:1021234607980

Gardner, B., de Bruijn, G., \& Lally, P. (2011). A systematic review and meta-analysis of applications of the self-report habit index to nutrition and physical activity behaviours. Annals of Behavioral Medicine, 42, 174-187. http://dx.doi. org/10.1007/s12160-011-9282-0

Gärling, T., Fujii, S., \& Boe, O. (2001). Empirical tests of a model of determinants of script-based driving choice. Transportation Research Part F, 4, 89-102. http://dx.doi.org/10.1016/S1369-8478(01)00016-X

Gärling, T., Gärling, A., \& Loukopoulos, P. (2002). Forecasting psychological consequences of car use reduction: A challenge to an environmental psychology of transportation. Applied Psychology: An International Review, 51(1), 90-106. http://dx.doi.org/10.1111/1464-0597.00080

Gollwitzer, P. M. (1999). Implementation intentions: Strong effects of simple plans. American Psychologist, 54(7), 493-503. http://dx.doi.org/10.1037/0003-066X.54.7.493

Haustein, S., Klöckner, Christian A., \& Blöbaum, A. (2009). Car use of young adults: The role of travel socialization. Transportation Research Part F, 12, 168-178. http://dx.doi.org/10.1016/j.trf.2008.10.003 
Instituto de Pesquisa Econômica Aplicada. (2011). Sistema de indicadores de percepção social: mobilidade urbana. Brasília, DF: Autor.

Klöckner, C. A., Matthies, E., \& Hunecke, M. (2003). Problems of operationalizing habits and integrating habits in normative decision-making models. Journal of Applied Social Psychology, 33(2), 396-417. http://dx.doi. org/10.1111/j.1559-1816.2003.tb01902.x

Klöckner, C. A., \& Verplanken, B. (2012). Yesterday's habits preventing change for tomorrow? About the influence of automaticity on environmental behavior. In L. Steg, A. E. van den Berg, \& J. I. M. de Groot (Eds.). Environmental psychology: An introduction (pp. 197-209). Hoboken: Wiley-Blackwell.

Lally, P., van Jaarsveld, C. H. M., Potts, H. W. W., \& Wardle, J. (2010). How are habits formed: Modelling habit formation in the real world. European Journal of Social Psychology, 40, 998-1009. http://dx.doi.org/10.1002/ejsp.674

Loibl, C., Kraybill, D. S., \& DeMay, S. W. (2011). Accounting for the role of habit in regular saving. Journal of Economic Psychology, 32, 581-592. http://dx.doi.org/10.1016/j.joep.2011.04.004

Mittal, B. (1988). Achieving higher seat belt usage: The role of habit in bridging the attitude-behavior gap. Journal of Applied Social Psychology, 18(12), 993-1016. http://dx.doi.org/10.1111/j.1559-1816.1988.tb01189.x

Neal, D. T., Wood, W., \& Quinn, J. M. (2006). Habits - A repeat performance. Current Directions in Psychological Science, 15(4), 198-202. http://dx.doi.org/10.1111/j.1467-8721.2006.00435.x

Neto, I. L., Feitosa, Z. O., Cristo, F., Cantal, C. B., \& Günther H. (2013). Uso de automóveis e qualidade de vida urbana: Desafios para a psicologia. Estudos de Psicologia (Natal), 18(4), 609-611. http://dx.doi.org/10.1590/S1413294X2013000400009

Ouellete, J. A., \& Wood, W. (1998). Habit and intention in everyday life: The multiple processes by which past behavior predicts future behavior. Psychological Bulletin, 124, 54-74. http://dx.doi.org/10.1037/0033-2909.124.1.54

Ronis, D. L., Yates, J. F., \& Kirscht, J. P. (1989). Attitudes, decisions, and habits as determinants of repeated behavior. In A. R. Pratkanis, S. J. Breckler \& A. G. Greenwald (Eds.). Attitude structure and function (pp. 213-239). Hillsdale, NJ: Lawrence Erlbaum Associates.

Steg, L., \& Vlek, C. (2009). Encouraging pro-environmental behaviour: An integrative review and research agenda. Journal of Environmental Psychology, 29, 309-317. http://dx.doi.org/10.1016/j.jenvp.2008.10.004

Verplanken, B. (2010). By force of habit. In A. Steptoe (Ed.). Handbook of behavioral medicine: Methods and applications (pp. 73-82). London: Springer. http://dx.doi.org/10.1007/978-0-387-09488-5_6

Verplanken, B., \& Aarts, H. (1999). Habit, attitude, and planned behaviour: Is habit an empty construct or an interesting case of goal-directed automaticity? European Review of Social Psychology, 10(1), 101-134. http://dx.doi. org/10.1080/14792779943000035

Verplanken, B., Aarts, H., van Knippenberg, A., \& Moonen, A. (1998). Habit versus planned behavior: A field experiment. British Journal of Social Psychology, 37, 111-128. http://dx.doi.org/10.1111/j.2044-8309.1998.tb01160.x

Verplanken, B., Myrbakk, V., \& Rudi, E. (2005). The measurement of habit. In T. Betsch \& S. Haberstroh (Eds.). The routines of decision making (pp. 231-247). Mahwah, NJ: Lawrence Erlbaum.

Verplanken, B., \& Orbell, S. (2003). Reflections on past behavior: A self-report index of habit strength. Journal of Applied Social Psychology, 33(6), 1313-1330. http://dx.doi.org/10.1111/j.1559-1816.2003.tb01951.x

Verplaken, B., \& Wood, W. (2006). Interventions to break and create consumer habits. Journal of Public Police \& Marketing, 25(1), 90-103. http://dx.doi.org/10.1509/jppm.25.1.90

Wittenbraker, J., Gibbs, B. L., \& Kahle, L. R. (1983). Seat belt attitudes, habits, and behaviors: An adaptive amendment to the Fishbein model. Journal of Applied Social Psychology, 13, 406-421. http://dx.doi.org/10.1111/j.1559-1816.1983. tb01748.x

Wood, W., Quinn, J. M., \& Kashy, D. (2002). Habits in everyday life: Thought, emotion, and action. Journal of Personality and Social Psychology, 83, 1281-1297. http://dx.doi.org/10.1037/0022-3514.83.6.1281

\footnotetext{
Autores:

HARTMUT GÜNTHER - Doutor, Universidade de Brasília.

Endereço para correspondência:

Fábio de Cristo

Rua 31 (Sul), lote 08, apto. 703-A, ed. Sedgwick

Águas Claras Sul

CEP 71929-720 Brasília, DF, Brasil

E-mail: <fabiodecristo@gmail.com>
}

FÁBIO DE CRISTO - Doutor, Universidade de Brasília e Centro Universitário de Brasília (UniCEUB).

Recebido em: 24.06 .14

Aceito em: 12.01 .15 